\title{
Downregulation of miR-200c protects cardiomyocytes from hypoxia-induced apoptosis by targeting GATA-4
}

\author{
ZHIGANG CHEN $^{1}$, SHAOLI ZHANG ${ }^{1}$, CHANGLEI GUO $^{1}$, JIANHUA LI $^{1}$ and WENFENG SANG ${ }^{2}$ \\ ${ }^{1}$ Department of Cardiology, The First Affiliated Hospital of Xinxiang Medical University, Weihui, \\ Henan 453100; ${ }^{2}$ Department of Internal Medicine Nursing, College of Nursing, \\ Xinxiang Medical University, Xinxiang, Henan 453003, P.R. China
}

Received November 21, 2016; Accepted March 22, 2017

DOI: $10.3892 /$ ijmm.2017.2959

\begin{abstract}
Hypoxia-induced cardiomyocyte apoptosis plays an important role in the development of ischemic heart disease. MicroRNAs (miRNAs or miRs) are emerging as critical regulators of hypoxia-induced cardiomyocyte apoptosis. miR-200c is an miRNA that has been reported to be related to apoptosis in various pathological processes; however, its role in hypoxia-induced cardiomyocyte apoptosis remains unclear. In the present study, we aimed to investigate the potential role and underlying mechanism of miR-200c in regulating hypoxia-induced cardiomyocyte apoptosis. We found that miR-200c was significantly upregulated by hypoxia in cardiomyocytes, as detected by reverse transcription-quantitative polymerase chain reaction (RT-qPCR). The lactate dehydrogenase, MTT, Annexin V/propidium iodide apoptosis and caspase-3 activity assays showed that downregulation of miR-200c markedly improved cell survival and suppressed the apoptosis of cardiomyocytes in response to hypoxia. Bioinformatics analysis and the dual-luciferase reporter assay demonstrated that miR-200c directly targeted the 3'-untranslated region of GATA-4, an important transcription factor for cardiomyocyte survival. RT-qPCR and western blot analysis showed that suppression of miR-200c significantly increased GATA-4 expression. Furthermore, downregulation of miR200c upregulated the expression of the anti-apoptotic gene $\mathrm{Bcl}-2$. However, the protective effects against hypoxia induced by the downregulation of miR-200c were significantly abolished by GATA-4 knockdown. Taken together, our results
\end{abstract}

Correspondence to: Professor Wenfeng Sang, Department of Internal Medicine Nursing, College of Nursing, Xinxiang Medical University, 601 Jinsui Avenue, Xinxiang, Henan 453003, P.R. China E-mail: sang_wenfeng@163.com

Abbreviations: miRNAs, microRNAs or miRs; RT-qPCR, reverse transcription-quantitative polymerase chain reaction; MTT, 3-(4,5dimethylthiazol-2-yl)-2,5-diphenyltetrazoliumbromide;UTR,untranslated region; $\mathrm{LDH}$, lactate dehydrogenase; PI, propidium iodide

Key words: cardiomyocytes, GATA-4, hypoxia, miR-200c suggest that downregulation of miR-200c protects cardiomyocytes from hypoxia-induced apoptosis by targeting GATA-4, providing a potential therapeutic molecular target for the treatment of ischemic heart disease.

\section{Introduction}

Ischemic heart disease represents one of the leading causes of death worldwide and has increased in incidence and prevalence in recent years (1). The mitochondria in cardiomyocytes are damaged in response to ischemia and hypoxia, leading to excessive apoptosis (2). The cardiomyocyte loss induced by apoptosis contributes to the development of ischemic heart disease $(3,4)$. Currently, an effective method for overcoming hypoxia-induced cardiomyocyte apoptosis is still lacking, due to the elusiveness of the underlying mechanism. Therefore, it is of great importance to gain a better understanding of the molecular mechanism underlying hypoxia-induced cardiomyocyte apoptosis, which may help to provide potential therapeutic approaches.

MicroRNAs (miRNAs or miRs) are a subset of endogenous, short and non-coding RNAs that negatively modulate gene expression $(5,6)$. miRNAs can impact the 3 '-untranslated region (3'-UTR) of target mRNAs, inducing mRNA degradation and translation inhibition $(5,6)$. Through modulation of gene expression in a post-transcriptional manner, miRNAs participate in various biological processes, including cell proliferation and apoptosis (7). A growing body of evidence suggests that miRNAs are involved in ischemic heart disease $(4,8,9)$, and they have been emerging as a new therapeutic strategy for this disease $(10,11)$. Targeting specific miRNAs has produced promising effects in inhibiting hypoxia-induced cardiomyocyte apoptosis (12-15). However, the precise effect of miRNAs on hypoxia-induced cardiomyocyte apoptosis requires further investigation.

GATA-4, a zinc-finger transcription factor, has been found to be an important regulator in cardiac development $(16,17)$. GATA-4 mediates cardiac hypertrophy by activating various genes including $\alpha$-myosin heavy chain, $\beta$-myosin heavy chain, myosin light chains, troponin I, troponin $\mathrm{C}$ and atrial natriuretic factor (18-20). Various hypertrophic stimuli, such as endothelin- 1 and $\alpha$-adrenergic agonist, can activate the expression of GATA- 4 in cardiomyocytes $(21,22)$. GATA-4 
also plays a critical role in regulating anti-apoptotic signaling in cardiomyocytes in response to hypoxic injury and myocardial ischemia or reperfusion injury $(23,24)$. GATA-4 inhibits doxorubicin-induced cardiomyocyte apoptosis by activating the anti-apoptotic gene, Bcl-2, in vitro and in vivo (23). GATA-4 has been suggested as a promising therapeutic target for the treatment of ischemic heart disease $(25,26)$.

miR-200c has been found to be an apoptosis-related miRNA in various pathological processes $(27,28)$ and increases in the gracilis muscle following ischemic injury (29). After ischemic preconditioning or focal cerebral ischemia, miR-200c was upregulated in the ischemic cortex (30). Inhibition of miR-200c was found to attenuate infarct volume and neurologic deficits in mice following cerebral ischemia (31). However, the role of miR-200c in ischemic heart disease is unclear. In this study, we aimed to investigate the potential role of miR-200c in ischemic heart disease using an in vitro model. We found that miR-200c was highly upregulated in cardiomyocytes exposed to hypoxia. Downregulation of miR-200c by transfection of an miR-200c inhibitor significantly reduced hypoxia-induced cardiomyocyte apoptosis and improved cell survival. Importantly, GATA-4 was identified as the target gene of miR-200c in cardiomyocytes. Downregulation of miR-200c increased the expression of GATA-4 and Bcl-2 in cardiomyocytes in response to hypoxia. Taken together, our results suggest that downregulation of miR-200c protects cardiomyocytes from hypoxia-induced apoptosis by targeting GATA-4, providing a potential therapeutic molecular target for the treatment of ischemic heart disease.

\section{Materials and methods}

Cell cultures. Rat cardiomyocyte cell line H9c2 and $293 \mathrm{~T}$ cells were both purchased from the American Type Culture Collection (ATCC; Manassas, VA, USA). Cells were grown in Dulbecco's modified Eagle's medium (DMEM) containing $10 \%$ fetal bovine serum (FBS) (both from Gibco, Rockville, MD, USA) and $1 \%$ penicillin-streptomycin solution (Sigma-Aldrich, St. Louis, MO, USA). Cells were routinely cultured in a humidified incubator containing $5 \% \mathrm{CO}_{2}$ at $37^{\circ} \mathrm{C}$. For the induction of hypoxia, $\mathrm{H} 9 \mathrm{c} 2$ cells were grown in a hypoxia chamber containing $94 \% \mathrm{~N}_{2}$, $5 \% \mathrm{CO}_{2}$ and $1 \% \mathrm{O}_{2}$ at $37^{\circ} \mathrm{C}$.

Cell transfection. The miR-200c mimics, miR-200c inhibitor and negative controls (NCs) were all purchased from GenePharma (Shanghai, China) and transfected into cells using Lipofectamine 2000 (Invitrogen Life Technologies, Carlsbad, CA, USA), following the manufacturer's instructions. The GATA-4 siRNA and NC siRNA were both purchased from Santa Cruz Biotechnology, Inc. (Santa Cruz, CA, USA) and transfected into cells according to the manufacturer's instructions.

Reverse transcription-quantitative polymerase chain reaction $(R T-q P C R)$. Total RNA was extracted using TRIzol reagent (Invitrogen Life Technologies) and reverse transcribed into cDNA using M-MLV Reverse Transcriptase (BioTeke Co., Ltd., Beijing, China) or TaqMan MicroRNA Reverse Transcription kit (Applied Biosystems, Foster City,
CA, USA). The cDNA was used as the template for RT-qPCR with SYBR-Green PCR Master Mix and appropriate primers on 7900HT Fast Real-Time PCR system (both from Applied Biosystems). U6 was used as the internal control for normalization of miR-200c. $\beta$-actin was used as the internal control for normalization of GATA-4 and Bcl-2. Relative gene expression was calculated by the $2^{-\Delta \Delta \mathrm{Cq}}$ method. The primer sequences used in the experiments were as follows: miR-200c forward, 5'-GTAATACTGCCGGGTAATGATGGA-3' and reverse, 5'-GTGCAGGGTCCGAGGT-3'; U6 forward, 5'-GCGCG TCGTGAAGCGTTC-3' and reverse, 5'-GTGCAGGGTCCG AGGT-3'; GATA-4 forward, 5'-GTGCCAACTGCCAGA CTACC-3' and reverse, 5'-AGCCTTGTGGGGACAGCTTC-3'; Bcl-2 forward, 5'-AGTTCGGTGGGGTCATGTGTG-3' and reverse, 5'-CCAGGTATGCACCCAGAGTG-3'; $\beta$-actin forward, 5'-TCAGGTCATCACTATCGGCAAT-3' and reverse, 5'-AAAGAAAGGGTGTAAAACGCA-3'.

3-(4,5-Dimethylthiazol-2-yl)-2,5-diphenyltetrazolium bromide (MTT) assay. Cell viability was assessed using the MTT colorimetric assay. In brief, the cells were seeded in 96-well plates at $2 \times 10^{4}$ cells/well and cultured overnight. The cells were transfected with the miR-200c inhibitor or NC inhibitor for $24 \mathrm{~h}$ and then subjected to hypoxic conditions for $24 \mathrm{~h}$. Afterwards, the cells were treated with $20 \mu \mathrm{l}$ of $5 \mathrm{mg} / \mathrm{ml}$ MTT (Sigma-Aldrich) and cultured for $4 \mathrm{~h}$. The purple-colored formazan crystals in living cells were solubilized by $200 \mu \mathrm{l}$ of dimethyl sulfoxide (DMSO; Sigma-Aldrich). After $15 \mathrm{~min}$, the absorbance of the solution at $490 \mathrm{~nm}$ was detected by a microplate reader (Bio-Rad Laboratories, Inc., Hercules, CA, USA).

Lactate dehydrogenase (LDH) assay. Cell injury was measured using an LDH assay kit (Roche Applied Science, Indianapolis, IN, USA) according to the manufacturer's instructions. The cells were lysed by $0.2 \%$ Triton X-100 (Sigma-Aldrich) and the supernatants were collected after centrifugation at $10,000 \mathrm{xg}$ for $10 \mathrm{~min}$ at $4^{\circ} \mathrm{C}$. The supernatants were then incubated with pyruvate and nicotinamide adenine dinucleotide hydrogen for $30 \mathrm{~min}$ at $37^{\circ} \mathrm{C}$. After the addition of $0.4 \mathrm{M} \mathrm{NaOH}$, the absorbance at $530 \mathrm{~nm}$ was detected by a microplate reader (Bio-Rad Laboratories, Inc.).

Annexin V/propidium iodide (PI) apoptosis assay. Cell apoptosis was measured by using an Annexin V/PI apoptosis detection kit (Beyotime Institute of Biotechnology, Haimen, China) following the manufacturer's recommended instructions. In conclusion, the cells were digested with $2.5 \mathrm{~g} / \mathrm{l}$ trypsin (Sigma-Aldrich) and then washed with phosphate-buffered saline (PBS). The cells were then re-suspended in binding buffer supplemented with $10 \mu \mathrm{l}$ of Annexin V. After incubation for $30 \mathrm{~min}, 5 \mu \mathrm{l}$ of PI solution was added and the cells were incubated for a further $5 \mathrm{~min}$. Cells were analyzed by flow cytometry (BD Biosciences, San Jose, CA, USA).

Caspase-3 activity assay. Caspase-3 activity was measured by a caspase-3 activity assay kit (Roche Applied Science), according to the manufacturer's instructions. In brief, the cells were lysed and the supernatants were collected after centrifugation at $16,000 \mathrm{xg}$ for $15 \mathrm{~min}$ at $4^{\circ} \mathrm{C}$. The supernatants were then incubated with $10 \mu \mathrm{l}$ Ac-DEVD-pNA $(2 \mathrm{mM})$ for $2 \mathrm{~h}$ 
at $37^{\circ} \mathrm{C}$. The absorbance at $405 \mathrm{~nm}$ was determined using a microplate reader (Bio-Rad Laboratories, Inc.).

Western blot analysis. Proteins were lysed in lysis buffer and protein concentrations were measured using a bicinchoninic acid assay kit (Beyotime Institute of Biotechnology). Equivalent amounts of proteins were loaded on $10 \%$ sodium dodecyl sulfate-polyacrylamide gels for separation, followed by protein transfer to a polyvinylidene fluoride membrane (Millipore, Bedford, MA, USA). The membrane was blocked with $5 \%$ non-fat dry milk for $1 \mathrm{~h}$ at $37^{\circ} \mathrm{C}$, followed by incubation with primary antibodies at $4^{\circ} \mathrm{C}$ overnight. The membrane was washed with Tris-buffered saline containing $0.1 \%$ Tween-20 (TBST) and then incubated with horseradish peroxidaseconjugated secondary antibodies (1:2,000; sc-2004; Santa Cruz Biotechnology, Inc.) for $1 \mathrm{~h}$ at $37^{\circ} \mathrm{C}$. After being washed with TBST, the protein bands were developed using a Pierce ECL Western Blotting kit (Pierce, Rockford, IL, USA). Gray values of protein bands were detected by Image-Pro Plus 6.0 software (Media Cybernetics, Inc., Rockville, MD, USA). The primary antibodies including anti-GATA-4 (1:500; sc-9053), anti-Bcl-2 $(1: 500 ;$ sc-783) and anti- $\beta$-actin $(1: 600 ; s c-130656)$ were all purchased from Santa Cruz Biotechnology, Inc.

Dual-luciferase reporter assay. The 3'-UTR of GATA-4 harboring either the miR-200c binding site (GATA-4 3'-UTR-WT) or a mutant (GATA-4 3'-UTR-MT) was cloned into the pmirGLO luciferase vector (Promega, Madison, WI, USA). The constructed vectors were co-transfected into 293T cells with the miR-200c inhibitor or NC inhibitor using Lipofectamine 2000 (Invitrogen Life Technologies,) and incubated for $48 \mathrm{~h}$. The cells were harvested and luciferase activities were measured using a Dual-GLO Luciferase Assay system (Promega).

Statistical analysis. The results are presented as mean \pm standard deviation. Statistical analyses were performed using SPSS package version 18.0 (SPSS, Inc., Chicago, IL, USA). Statistical significance was determined by one-way analysis of variance (ANOVA) followed by a Bonferroni correction. Differences were regarded as statistically significant at values of $\mathrm{p}<0.05$.

\section{Results}

miR-200c is upregulated by hypoxia in cardiomyocytes. To investigate the possible role of miR-200c in ischemic heart disease, we detected the expression of miR-200c in cardiomyocytes exposed to hypoxia using RT-qPCR. The results showed that miR-200c was significantly upregulated after exposure to hypoxia in comparison to the control (Fig. 1A), implying that miR-200c has an important role in the hypoxic injury of cardiomyocytes.

Downregulation of miR-200c attenuates hypoxia-induced cell injury. To investigate the biological effect of miR-200c on hypoxia-treated cardiomyocytes, we inhibited the expression of miR-200c in cells by transfecting them with an miR-200c inhibitor (Fig. 1B). We then detected the effect of miR-200c suppression on cell viability with the MTT assay.

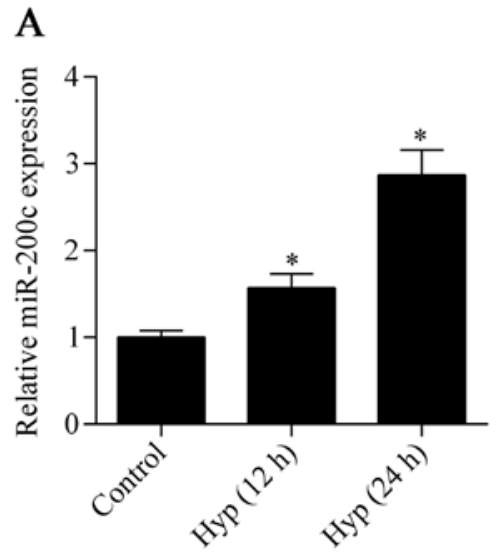

B

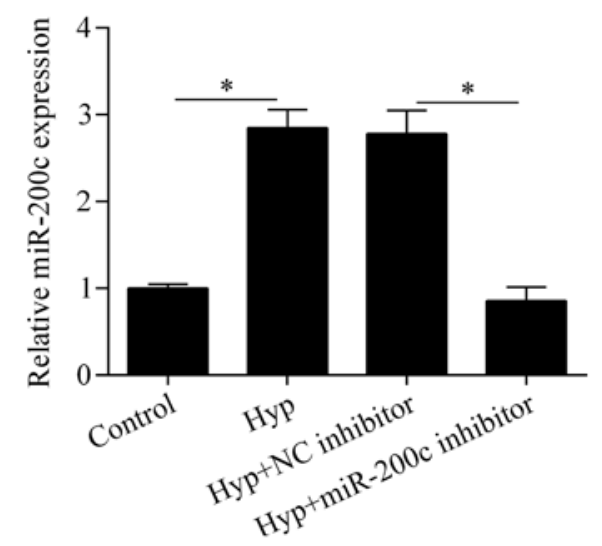

Figure 1. Expression of miR-200c in cardiomyocytes. (A) The expression of miR-200c in H9c2 cells under hypoxic conditions for 12 and $24 \mathrm{~h}$ as detected by RT-qPCR. Hyp, hypoxia. Cells cultured under normoxic conditions were used as the control. "p<0.05 vs. control. (B) The expression of miR-200c in miR-200c inhibitor-transfected H9c2 cells. H9c2 cells were transfected with miR-200c inhibitor or NC inhibitor for $24 \mathrm{~h}$ and subjected to hypoxia for 24 h. ${ }^{*} \mathrm{p}<0.05, \mathrm{n}=3$ for each group. Three independent experiments were performed.

The results showed that cell viability was markedly impaired by hypoxia but was partially improved by miR-200c inhibition (Fig. 2A). We next evaluated the effect of miR-200c inhibition on hypoxia-induced cell injury with the LDH assay. We found that the hypoxia-induced cell injury was also significantly reversed by the downregulation of miR200c (Fig. 2B). Taken together, these data suggest that downregulation of miR-200c improves cell survival under hypoxic conditions.

Downregulation of $\mathrm{miR}-200 \mathrm{c}$ inhibits cardiomyocyte apoptosis induced by hypoxia. To verify the protective effect of miR-200c inhibition on cardiomyocytes under hypoxia, we further investigated the effect of miR-200c inhibition on hypoxia-induced apoptosis. The Annexin V/PI apoptosis assay showed that hypoxia-induced apoptosis was significantly suppressed by the downregulation of miR-200c (Fig. 3A and B). Furthermore, the increased activity of the pro-apoptotic protein, caspase-3, induced by hypoxia was also markedly decreased by miR-200c inhibition (Fig. 3C). Overall, these results indicate that the downregulation of miR-200c suppresses hypoxia-induced cardiomyocyte apoptosis. 
A

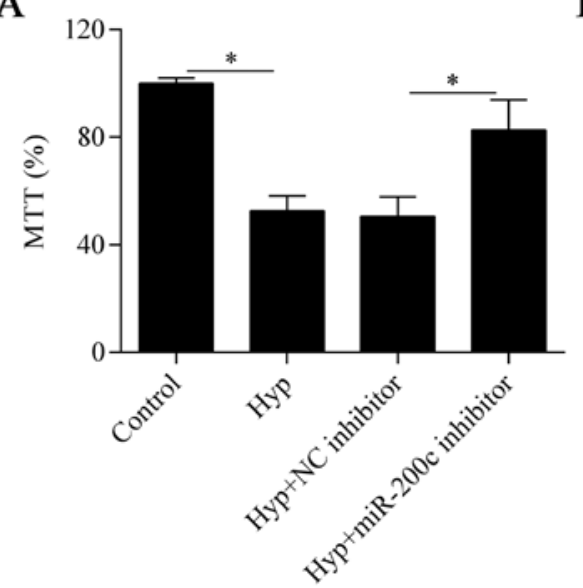

B

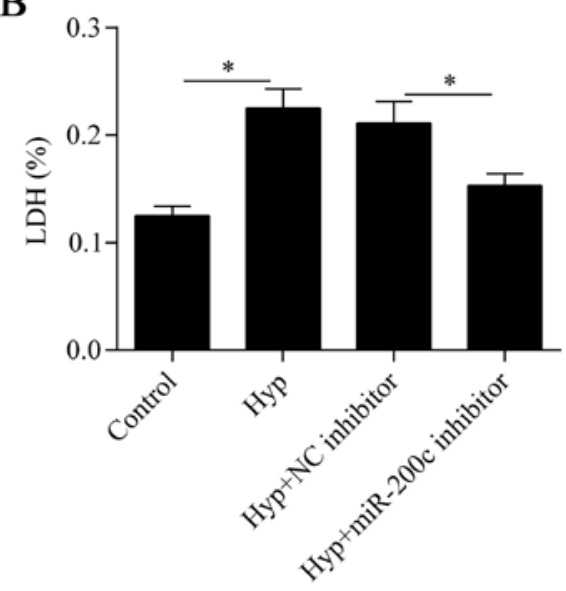

Figure 2. Downregulation of miR-200c attenuates hypoxia-induced cell injury. H9c2 cells were transfected with an miR-200c inhibitor or NC inhibitor for $24 \mathrm{~h}$ and subjected to hypoxia for $24 \mathrm{~h}$. (A) Cell viability was detected by the MTT assay. (B) Cell injury was detected by the lactate dehydrogenase (LDH) assay. * $\mathrm{p}<0.05, \mathrm{n}=3$ for each group. Three independent experiments were performed. Hyp, hypoxia.

A

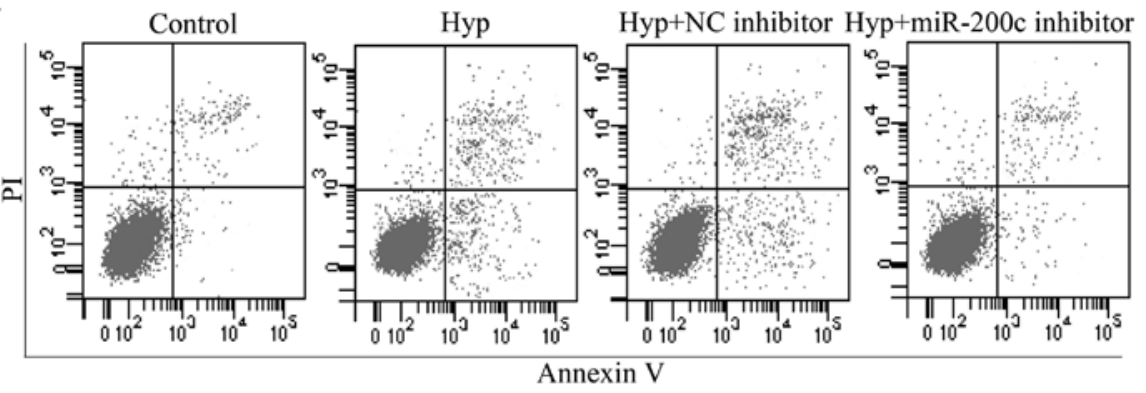

B

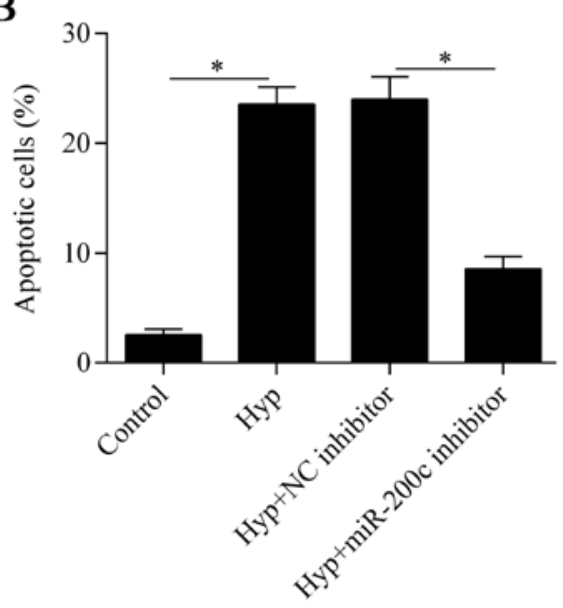

$\mathrm{C}$

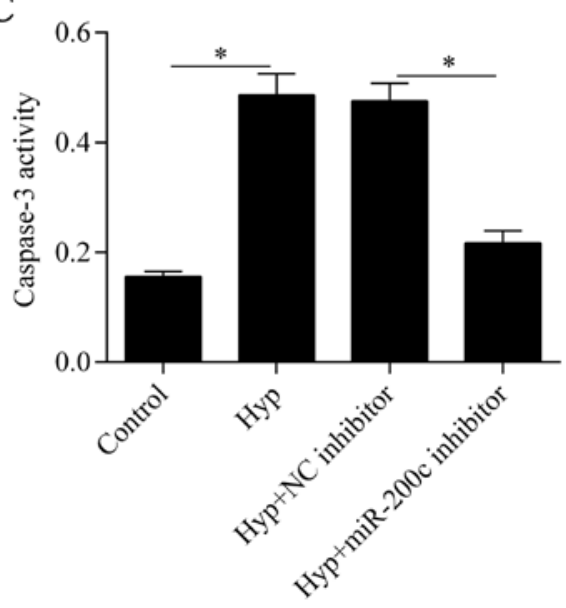

Figure 3. Downregulation of miR-200c inhibits hypoxia-induced cardiomyocyte apoptosis. H9c2 cells were transfected with an miR-200c inhibitor or NC inhibitor for $24 \mathrm{~h}$ and subjected to hypoxia for $24 \mathrm{~h}$. (A) Cell apoptosis was examined with the Annexin V/PI apoptosis assay. (B) Quantitative data of Annexin V/PI apoptosis assay. (C) Caspase-3 activity was measured by the caspase-3 activity assay. * $\mathrm{p}<0.05$, $\mathrm{n}=3$ for each group. Three independent experiments were performed. Hyp, hypoxia.

GATA-4 is a potential target of miR-200c. To investigate the underlying mechanism by which the suppression of miR-200c provides a protective effect against hypoxia, we predicted the target genes of miR-200c with bioinformatics analysis. We found that GATA-4, an important transcription factor for cardiomyocyte survival $(23,24)$, was a potential target gene of miR-200c. The 3'-UTR of GATA-4, harboring the complementary seed-matched binding sites of the miR-200c binding site (GATA-4 3'-UTR-WT) and a mutant (GATA-4 3'-UTR-MT), are shown in Fig. 4A. To confirm the presence of the interaction between miR-200c and GATA-4 3'-UTR, GATA-4 3'-UTR-WT or GATA-4 3'-UTR-MT was cloned into the pmirGLO vector that was used for the luciferase reporter assay. The results showed that the luciferase 
A

3'-GUAGUAAUGGGCCGUCAUAAU-5' Rno-miR-200c | | | | | |

5'-AUGGCUGUAAAAUCAGUAUUU-3'GATA-43'-UTR-WT

5'-AUGGCUGUAAAAUGACUUUAU-3'GATA-4 3'-UTR-MT

B

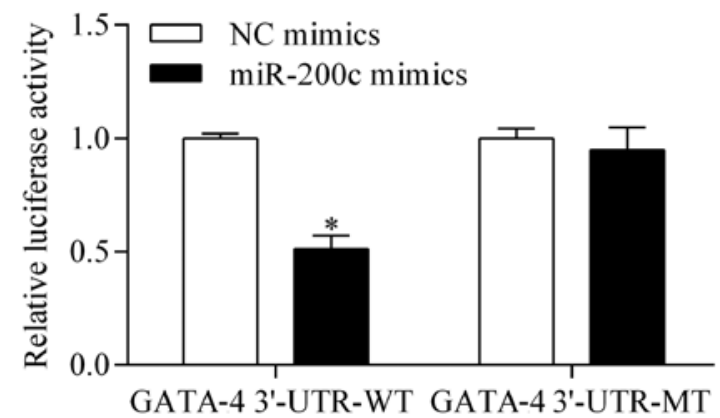

Figure 4. miR-200c targets the 3'-untranslated region (3'-UTR) of GATA-4. (A) Schematic diagram of the binding sites between miR-200c and GATA-4 3'-UTR. (B) The effect of miR-200c on luciferase activity was detected by the dual-luciferase reporter assay. The pmirGLO-GATA-4 3'-UTR-WT or pmirGLO-GATA-4 3'-UTR-MT was co-transfected with the miR-200c inhibitor or NC inhibitor into $293 \mathrm{~T}$ cells. ${ }^{*} \mathrm{p}<0.05$, compared with the NC mimics, $n=3$ for each group. Three independent experiments were performed.

activity of pmirGLO-GATA-4 3'-UTR-WT was significantly decreased by miR-200c overexpression (Fig. 4B). However, the luciferase activity of pmirGLO-GATA-4 3'-UTR-MT was not obviously affected by miR-200c overexpression (Fig. 4B). These results suggest that miR-200c directly targets the 3'-UTR of GATA-4. We then examined the direct effect of miR-200c on GATA-4 expression by RT-qPCR and western blot analysis. The results showed that the downregulation of miR-200c significantly increased the mRNA (Fig. 5A) and protein (Fig. 5B) expression of GATA-4, which were decreased by hypoxia in the cardiomyocytes. Taken together, these results suggest that GATA-4 is a direct target gene of miR-200c in cardiomyocytes.

Downregulation of $\mathrm{miR}-200$ c promotes the expression of $B c l-2$. To further investigate the molecular basis of miR-200c in regulating cardiomyocyte survival, we detected the expression of the anti-apoptotic gene $\mathrm{Bcl}-2$, a downstream gene of GATA-4 (23). The results showed that the downregulation of miR-200c significantly upregulated the mRNA (Fig. 6A) and protein (Fig. 6B) expression of $\mathrm{Bcl}-2$ in response to hypoxia, implying that miR-200c regulates Bcl-2 expression.

Knockdown of GATA-4 abolishes the protective effect of $m i R-200 c$ inhibition. To verify that GATA-4 contributes to the miR-200c inhibition-mediated protective effect against hypoxia, we silenced the expression of GATA-4 at the same time as suppressing miR-200c. The results showed that the promotive effect of miR-200c suppression on GATA-4 expression was significantly abolished by the knockdown of GATA-4 (Fig. 7A). Similarly, the increased expression of Bcl-2 induced by miR-200c suppression was eliminated by the knockdown of GATA-4 (Fig. 7B). As expected, the protective effect of miR-200c suppression against hypoxia was also markedly reversed by the knockdown of GATA-4 (Fig. 7C and D).

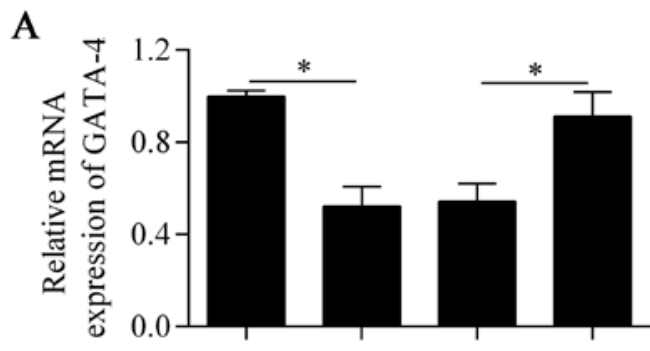

B

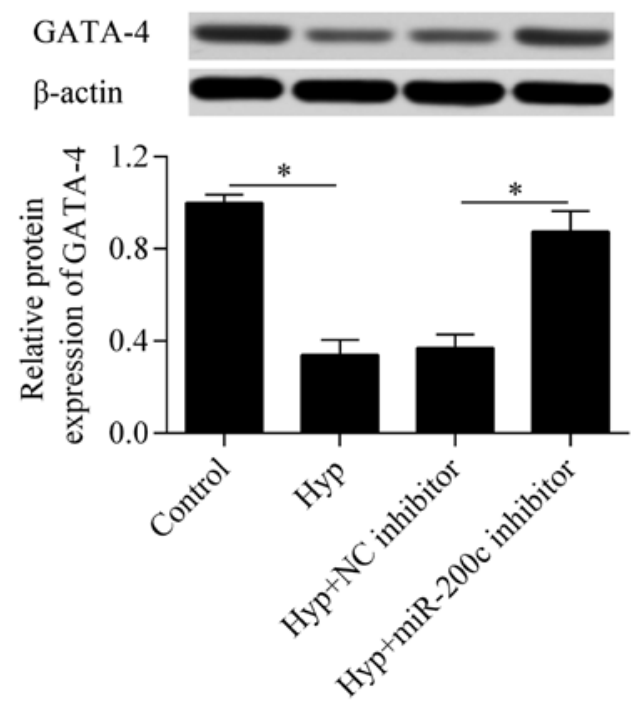

Figure 5. Downregulation of miR-200c increases GATA-4 expression. $\mathrm{H} 9 \mathrm{c} 2$ cells were transfected with the miR-200c inhibitor or NC inhibitor for $24 \mathrm{~h}$ and subjected to hypoxia for $24 \mathrm{~h}$. (A) The mRNA expression of GATA-4 was detected by RT-qPCR. (B) The protein expression of GATA-4 was detected by western blot analysis. ${ }^{*} \mathrm{p}<0.05, \mathrm{n}=3$ for each group. Three independent experiments were performed. Hyp, hypoxia.

Taken together, these results suggest that downregulation of miR-200c protects cardiomyocytes against hypoxia through the promotion of GATA-4 expression.

\section{Discussion}

Overcoming hypoxia-induced cardiomyocyte apoptosis is a main obstacle for the treatment of ischemic heart disease. In this study, we report that miR-200c is a novel regulator of hypoxia-induced cardiomyocyte apoptosis. We found that miR-200c was highly upregulated in cardiomyocytes subjected to hypoxia treatment. Our data demonstrated that the downregulation of miR-200c provided protective effects against hypoxia in cardiomyocytes by upregulating GATA-4 and $\mathrm{Bcl}-2$ expression, indicating a potential therapeutic target for ischemic heart disease.

Numerous studies have demonstrated that miR-200c induces the apoptosis of various types of cancer cells $(27,32,33)$. miR-200c sensitizes tumor cells to apoptosis by targeting Fas-associated phosphatase-1 (34). Similarly, miR-200c was significantly increased after spinal cord injury and reactive oxygen species were found to enhance miR-200c expression, which induced apoptosis in microglial cells (34). In endothelial cells, miR-200c overexpression induced by reactive oxygen 
A

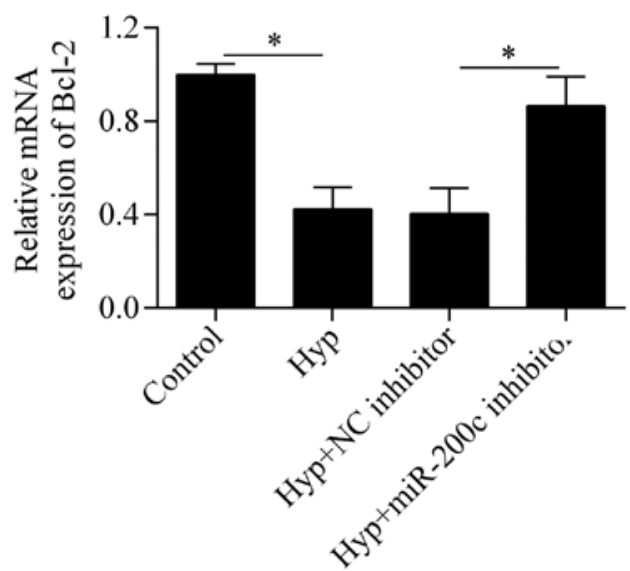

B
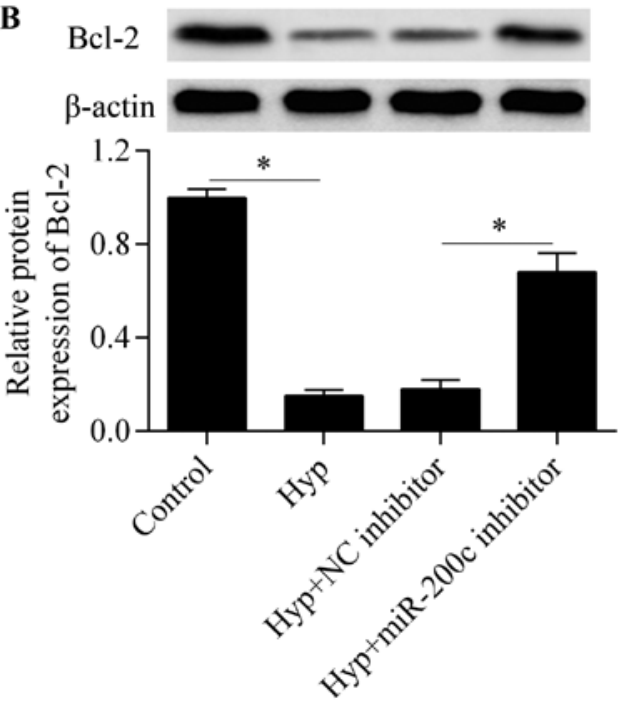

Figure 6. Downregulation of miR-200c promotes the expression of Bcl-2. H9c2 cells were transfected with the miR-200c inhibitor or NC inhibitor for $24 \mathrm{~h}$ and subjected to hypoxia for $24 \mathrm{~h}$. (A) The mRNA expression of Bcl-2 was detected by RT-qPCR. (B) The protein expression of Bcl-2 was detected by western blot analysis. ${ }^{*}<0.05, \mathrm{n}=3$ for each group. Three independent experiments were performed. Hyp, hypoxia.

A

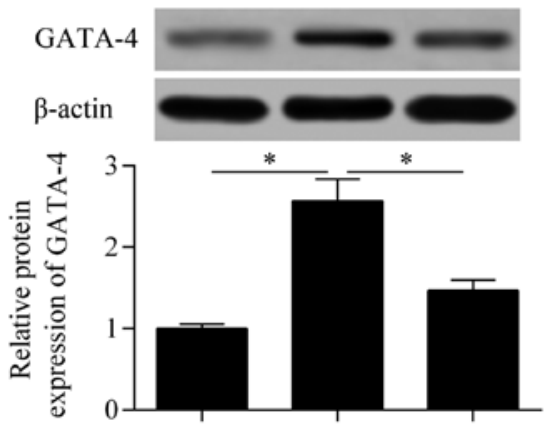

C

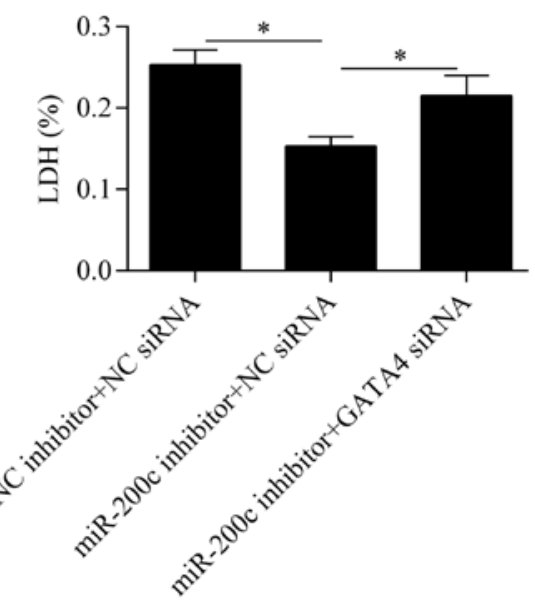

B

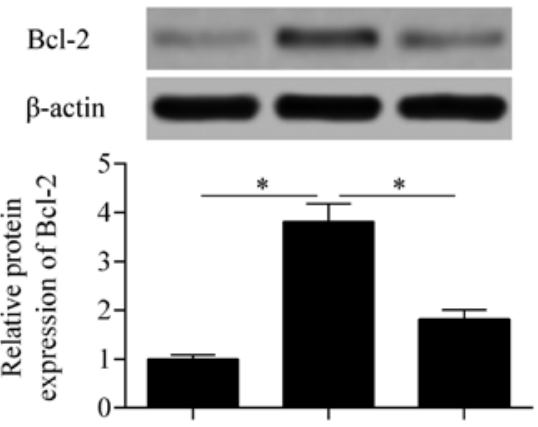

D

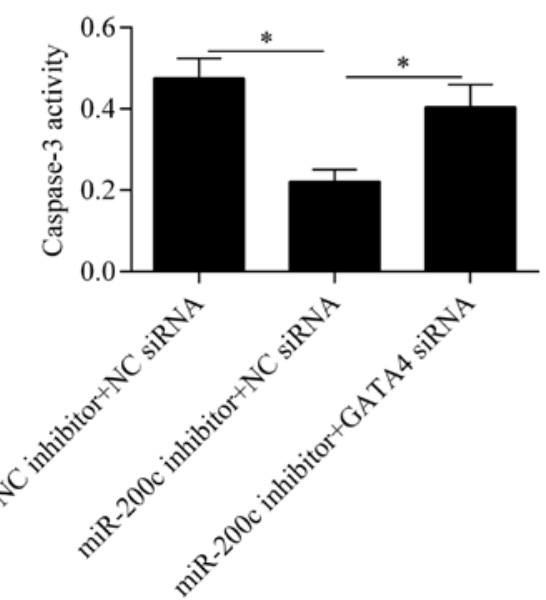

Figure 7. GATA-4 knockdown abolishes the effects of miR-200c suppression. H9c2 cells were co-transfected with the miR-200c inhibitor and GATA-4 siRNA for $24 \mathrm{~h}$, and then exposed to hypoxia for $24 \mathrm{~h}$. The protein expression of (A) GATA-4 and (B) Bcl-2 was detected by western blot analysis. (C) Cell injury was detected by the LDH assay. (D) Cell apoptosis was measured by the caspase- 3 activity assay. ${ }^{*} \mathrm{p}<0.05$, $\mathrm{n}=3$ for each group. Three independent experiments were performed.

species promoted cell growth arrest, senescence and apoptosis by targeting zinc-finger E-box binding homeobox 1 (35). Downregulation of miR-200c preserved endothelial function in diabetic mice by targeting zinc-finger E-box binding homeobox 1 and inhibiting COX-2 (36). miR-200c was found to increase in the gracilis muscle following ischemic injury (29). The inhibition of miR-200c attenuated hepatic ischemia or reperfusion injury (37). Moreover, miR-200c was found to be 
upregulated in the ischemic cortex after ischemic preconditioning or focal cerebral ischemia (30). Downregulation of miR-200c attenuated infarct volume and neurologic deficit in mice following cerebral ischemia by targeting Reelin (31), and inhibited cardiomyocyte hypertrophy in high glucose-treated cardiomyocytes (38). However, no data have indicated the role of miR-200c in regulating cardiomyocyte apoptosis. In this study, we found that miR-200c was highly upregulated by hypoxia in cardiomyocytes. The downregulation of miR200c suppressed hypoxia-induced cardiomyocyte apoptosis, indicating an important role of miR-200c in regulating cardiomyocyte survival in response to stress injury.

To investigate the potential mechanism underlying the regulation of cardiomyocyte apoptosis by miR-200c, we aimed to identify the potential target gene of miR-200c in cardiomyocytes. We identified GATA-4, an important transcriptional factor for cardiomyocyte survival $(23,24)$, to be a target gene of miR-200c. GATA-4 was found to be suppressed by anthracyclines in cardiomyocytes and the restoration of GATA-4 attenuated cardiomyocyte apoptosis (39). Activation of GATA-4 promoted cell survival and reduced cell death induced by daunorubicin (40) and doxorubicin (41). GATA-4 can activate the anti-apoptotic signaling in cardiomyocytes and protect cardiomyocytes against hypoxic injury and myocardial ischemia or reperfusion injury $(23,24)$. GATA-4 has becoming a promising therapeutic target for the treatment of ischemic heart disease $(25,26)$. The co-culture of GATA-4 gene-engineered mesenchymal stem cells and cardiomyocytes inhibited hypoxia-induced apoptosis $(42,43)$. Moreover, exosomes derived from the overexpression of GATA-4 in mesenchymal stem cells also showed cardioprotection (44). It has been reported that GATA-4 inhibits cardiomyocyte apoptosis by activating Bcl-2 (23). Kobayashi et al found that GATA-4 directly binds to the GATA site in the promoter of Bcl-2 and positively regulated Bcl-2 expression in cardiomyocytes in vivo and in vitro (23). A study also showed that GATA-4 regulated Bcl-2 expression in ovarian granulosa cell tumors (45). Cardiac ankyrin repeat protein has been reported to repress cardiomyocyte apoptosis induced by hypoxia or reoxygenation by binding the Bcl-2 promoter by interacting with GATA-4 (46). These findings suggest that GATA-4 can serve as a promising therapeutic target for preventing cardiomyocyte apoptosis. In this study, we found that GATA-4 is a target gene of miR-200c. Downregulation of miR-200c promoted the expression of GATA-4, thus protecting cardiomyocytes from hypoxia-induced apoptosis. Furthermore, silencing of GATA-4 abolished the miR-200c inhibition-induced protective effects.

The activity of GATA-4 is controlled by various post-translational modifications including ubiquitination, phosphorylation and acetylation $(20,47)$. Hypoxia induces the ubiquitination of GATA-4 and the attenuation of GATA-4 ubiquitination by erythropoietin increases cell viability under hypoxia (48). Recent studies also showed that GATA-4 undergoes epigenetic regulation by miRNAs $(49,50)$. Overexpression of miR-26b was found to suppress GATA-4 expression by targeting the 3'-UTR, leading to increased cardiomyocyte apoptosis (51). miR-26a attenuated cardiac hypertrophy via the targeting of GATA-4 in cultured cardiomyocytes (52). Downregulation of miR-208a suppressed doxorubicin-induced cardiomyocyte apoptosis by promoting GATA-4 (53). In this study, we found that miR-200c also targeted and regulated GATA-4 in cardiomyocytes. The inhibition of miR-200c protected cardiomyocytes against hypoxia-induced apoptosis by targeting GATA-4. In line with our findings, miR-200c was found to regulate embryonic stem cell renewal and differentiation by targeting GATA-4 (54). Our study indicates that miR-200c may serve as a promising target for the development of miRNA-based therapy for ischemic heart disease by targeting GATA-4.

In conclusion, our study demonstrated that miR-200c is a hypoxia-response gene in cardiomyocytes and is induced by hypoxia. Downregulation of miR-200c provides considerable protective effects against hypoxia in cardiomyocytes by promoting GATA-4 and Bcl-2 expression. Our study suggests a potential therapeutic molecular target for the treatment of ischemic heart disease. However, the precise role of miR-200c and GATA-4 in regulating cardiomyocyte apoptosis remains to be fully elucidated in vivo using animal models.

\section{References}

1. Yellon DM and Hausenloy DJ: Myocardial reperfusion injury. N Engl J Med 357: 1121-1135, 2007.

2. Glinka YY and Youdim MB: Inhibition of mitochondrial complexes I and IV by 6-hydroxydopamine. Eur J Pharmacol 292: 329-332, 1995.

3. Kitsis RN, Peng CF and Cuervo AM: Eat your heart out. Nat Med 13: 539-541, 2007.

4. Kang PM, Haunstetter A, Aoki H, Usheva A and Izumo S: Morphological and molecular characterization of adult cardiomyocyte apoptosis during hypoxia and reoxygenation. Circ Res 87: 118-125, 2000.

5. Filipowicz W, Bhattacharyya SN and Sonenberg N: Mechanisms of post-transcriptional regulation by microRNAs: are the answers in sight? Nat Rev Genet 9: 102-114, 2008.

6. Winter J, Jung S, Keller S, Gregory RI and Diederichs S: Many roads to maturity: microRNA biogenesis pathways and their regulation. Nat Cell Biol 11: 228-234, 2009.

7. Su Z, Yang Z, Xu Y, Chen Y and Yu Q: MicroRNAs in apoptosis, autophagy and necroptosis. Oncotarget 6: 8474-8490, 2015.

8. Wang Y, Pan X, Fan Y, Hu X, Liu X, Xiang M and Wang J: Dysregulated expression of microRNAs and mRNAs in myocardial infarction. Am J Transl Res 7: 2291-2304, 2015.

9. Boon RA and Dimmeler S: MicroRNAs in myocardial infarction. Nat Rev Cardiol 12: 135-142, 2015.

10. Hang P, Guo J, Sun C and Du Z: MicroRNAs as candidate drug targets for cardiovascular diseases. Curr Drug Targets: Feb 29, 2016 (Epub ahead of print).

11. Samanta S, Balasubramanian S, Rajasingh S, Patel U, Dhanasekaran A, Dawn B and Rajasingh J: MicroRNA: a new therapeutic strategy for cardiovascular diseases. Trends Cardiovasc Med 26: 407-419, 2016.

12. Zou Y, Liu W, Zhang J and Xiang D: miR-153 regulates apoptosis and autophagy of cardiomyocytes by targeting Mcl-1. Mol Med Rep 14: 1033-1039, 2016.

13. Xu H, Jin L, Chen Y and Li J: Downregulation of microRNA-429 protects cardiomyocytes against hypoxia-induced apoptosis by increasing Notch1 expression. Int J Mol Med 37: 1677-1685, 2016.

14. Xie J, Hu X, Yi C, Hu G, Zhou X and Jiang H: MicroRNA 451 protects against cardiomyocyte anoxia/reoxygenation injury by inhibiting high mobility group box 1 expression. Mol Med Rep 13: 5335-5341, 2016.

15. Ke ZP, Xu P, Shi Y and Gao AM: MicroRNA-93 inhibits ischemia-reperfusion induced cardiomyocyte apoptosis by targeting PTEN. Oncotarget 7: 28796-28805, 2016.

16. Kelley C, Blumberg H, Zon LI and Evans T: GATA-4 is a novel transcription factor expressed in endocardium of the developing heart. Development 118: 817-827, 1993.

17. Arceci RJ, King AA, Simon MC, Orkin SH and Wilson DB: Mouse GATA-4: a retinoic acid-inducible GATA-binding transcription factor expressed in endodermally derived tissues and heart. Mol Cell Biol 13: 2235-2246, 1993. 
18. Liang Q, De Windt LJ, Witt SA, Kimball TR, Markham BE and Molkentin JD: The transcription factors GATA4 and GATA6 regulate cardiomyocyte hypertrophy in vitro and in vivo. J Biol Chem 276: 30245-30253, 2001

19. Charron F, Tsimiklis G, Arcand M, Robitaille L, Liang Q, Molkentin JD, Meloche S and Nemer M: Tissue-specific GATA factors are transcriptional effectors of the small GTPase RhoA. Genes Dev 15: 2702-2719, 2001.

20. Suzuki YJ: Cell signaling pathways for the regulation of GATA4 transcription factor: implications for cell growth and apoptosis. Cell Signal 23: 1094-1099, 2011

21. Morimoto T, Hasegawa K, Kaburagi S, Kakita T, Wada H, Yanazume T and Sasayama S: Phosphorylation of GATA-4 is involved in alpha 1-adrenergic agonist-responsive transcription of the endothelin-1 gene in cardiac myocytes. J Biol Chem 275: 13721-13726, 2000

22. Hautala N, Tokola H, Luodonpää M, Puhakka J, Romppanen $H$, Vuolteenaho $\mathrm{O}$ and Ruskoaho H: Pressure overload increases GATA4 binding activity via endothelin-1. Circulation 103: 730-735, 2001.

23. Kobayashi S, Lackey T, Huang Y, Bisping E, Pu WT, Boxer LM and Liang Q: Transcription factor gata4 regulates cardiac BCL2 gene expression in vitro and in vivo. FASEB J 20: 800-802, 2006.

24. Kim Y, Ma AG, Kitta K, Fitch SN, Ikeda T, Ihara Y, Simon AR, Evans T and Suzuki YJ: Anthracycline-induced suppression of GATA-4 transcription factor: implication in the regulation of cardiac myocyte apoptosis. Mol Pharmacol 63: 368-377, 2003

25. Rysä J, Tenhunen O, Serpi R, Soini Y, Nemer M, Leskinen H and Ruskoaho H: GATA-4 is an angiogenic survival factor of the infarcted heart. Circ Heart Fail 3: 440-450, 2010.

26. Bian J, Popovic ZB, Benejam C, Kiedrowski M, Rodriguez LL and Penn MS: Effect of cell-based intercellular delivery of transcription factor GATA4 on ischemic cardiomyopathy. Circ Res 100: 1626-1633, 2007.

27. Shan W, Zhang X, Li M, Deng F and Zhang J: Over expression of miR-200c suppresses invasion and restores methotrexate sensitivity in lung cancer A549 cells. Gene 593: 265-271, 2016.

28. Boominathan L: The tumor suppressors p53, p63, and p73 are regulators of microRNA processing complex. PLoS One 5 : e10615, 2010.

29. Hsieh CH, Jeng JC, Jeng SF, Wu CJ, Lu TH, Liliang PC, Rau CS, Chen YC and Lin CJ: MicroRNA profiling in ischemic injury of the gracilis muscle in rats. BMC Musculoskelet Disord 11: $123,2010$.

30. Lee ST, Chu K, Jung KH, Yoon HJ, Jeon D, Kang KM, Park KH, Bae EK, Kim M, Lee SK, et al: MicroRNAs induced during ischemic preconditioning. Stroke 41: 1646-1651, 2010.

31. Stary CM, Xu L, Sun X, Ouyang YB, White RE, Leong J, Li J, Xiong X and Giffard RG: MicroRNA-200c contributes to injury from transient focal cerebral ischemia by targeting Reelin. Stroke 46: 551-556, 2015.

32. Venkatadri R, Muni T, Iyer AK, Yakisich JS and Azad N: Role of apoptosis-related miRNAs in resveratrol-induced breast cancer cell death. Cell Death Dis 7: e2104, 2016.

33. Cui J, Cheng Y, Zhang P, Sun M, Gao F, Liu C and Cai J: Downregulation of miR200c promotes radiation-induced thymic lymphoma by targeting BMI1. J Cell Biochem 115: $1033-1042,2014$

34. Yu DS, Lv G, Mei XF, Cao Y, Wang YF, Wang YS and Bi YL: MiR-200c regulates ROS-induced apoptosis in murine BV-2 cells by targeting FAP-1. Spinal Cord: Dec 2, 2014 (Epub ahead of print).

35. Magenta A, Cencioni C, Fasanaro P, Zaccagnini G, Greco S, Sarra-Ferraris G, Antonini A, Martelli F and Capogrossi MC: miR-200c is upregulated by oxidative stress and induces endothelial cell apoptosis and senescence via ZEB1 inhibition. Cell Death Differ 18: 1628-1639, 2011.

36. Zhang H, Liu J, Qu D, Wang L, Luo JY, Lau CW, Liu P, Gao Z, Tipoe GL, Lee HK, et al: Inhibition of miR-200c restores endothelial function in diabetic mice through suppression of COX-2. Diabetes 65: 1196-1207, 2016.
37. Wu Y, Gu C and Huang X: Sevoflurane protects against hepatic ischemia/reperfusion injury by modulating microRNA-200c regulation in mice. Biomed Pharmacother 84: 1126-1136, 2016.

38. Singh GB, Raut SK, Khanna S, Kumar A, Sharma S, Prasad R and Khullar M: MicroRNA-200c modulates DUSP-1 expression in diabetes-induced cardiac hypertrophy. Mol Cell Biochem 424: 1-11, 2017.

39. Suzuki YJ and Evans T: Regulation of cardiac myocyte apoptosis by the GATA-4 transcription factor. Life Sci 74: 1829-1838, 2004

40. Suzuki YJ: Stress-induced activation of GATA-4 in cardiac muscle cells. Free Radic Biol Med 34: 1589-1598, 2003.

41. Kobayashi S, Volden P, Timm D, Mao K, Xu X and Liang Q: Transcription factor GATA4 inhibits doxorubicin-induced autophagy and cardiomyocyte death. J Biol Chem 285: 793-804, 2010

42. Li HX, Zhou YF, Zhao X, Jiang B and Yang XJ: GATA-4 protects against hypoxia-induced cardiomyocyte injury: effects on mitochondrial membrane potential. Can J Physiol Pharmacol 92: 669-678, 2014

43. Yu B, Gong M, Wang Y, Millard RW, Pasha Z, Yang Y, Ashraf M and Xu M: Cardiomyocyte protection by GATA-4 gene engineered mesenchymal stem cells is partially mediated by translocation of miR-221 in microvesicles. PLoS One 8: e73304, 2013.

44. Yu B, Kim HW, Gong M, Wang J, Millard RW, Wang Y, Ashraf $\mathrm{M}$ and $\mathrm{Xu} \mathrm{M}$ : Exosomes secreted from GATA-4 overexpressing mesenchymal stem cells serve as a reservoir of anti-apoptotic microRNAs for cardioprotection. Int J Cardiol 182: 349-360, 2015.

45. Kyrönlahti A, Rämö M, Tamminen M, Unkila-Kallio L, Butzow R, Leminen A, Nemer M, Rahman N, Huhtaniemi I, Heikinheimo $\mathrm{M}$, et al: GATA-4 regulates Bcl-2 expression in ovarian granulosa cell tumors. Endocrinology 149: 5635-5642, 2008

46. Zhang N, Ye F, Zhu W, Hu D, Xiao C, Nan J, Su S, Wang Y, Liu M, Gao K, et al: Cardiac ankyrin repeat protein attenuates cardiomyocyte apoptosis by upregulation of $\mathrm{Bcl}-2$ expression. Biochim Biophys Acta 1863: 3040-3049, 2016.

47. Pikkarainen S, Tokola H, Kerkelä R and Ruskoaho H: GATA transcription factors in the developing and adult heart. Cardiovase Res 63: 196-207, 2004.

48. Jun JH, Shin EJ, Kim JH, Kim SO, Shim JK and Kwak YL: Erythropoietin prevents hypoxia-induced GATA-4 ubiquitination via phosphorylation of serine 105 of GATA-4. Biol Pharm Bull 36: 1126-1133, 2013.

49. Yao CX, Wei QX, Zhang YY, Wang WP, Xue LX, Yang F, Zhang SF, Xiong CJ, Li WY, Wei ZR, et al: miR-200b targets GATA-4 during cell growth and differentiation. RNA Biol 10: 465-480, 2013.

50. Sowa N, Horie T, Kuwabara Y, Baba O, Watanabe S, Nishi H, Kinoshita M, Takanabe-Mori R, Wada H, Shimatsu A, et al: MicroRNA 26b encoded by the intron of small CTD phosphatase (SCP) 1 has an antagonistic effect on its host gene. J Cell Biochem 113: 3455-3465, 2012.

51. Han M, Yang Z, Sayed D, He M, Gao S, Lin L, Yoon S and Abdellatif M: GATA4 expression is primarily regulated via a miR-26b-dependent post-transcriptional mechanism during cardiac hypertrophy. Cardiovasc Res 93: 645-654, 2012.

52. Liu Y, Wang Z and Xiao W: MicroRNA-26a protects against cardiac hypertrophy via inhibiting GATA4 in rat model and cultured cardiomyocytes. Mol Med Rep 14: 2860-2866, 2016.

53. Tony H, Yu K and Qiutang Z: MicroRNA-208a silencing attenuates doxorubicin induced myocyte apoptosis and cardiac dysfunction. Oxid Med Cell Longev 2015: 597032, 2015.

54. Huang HN, Chen SY, Hwang SM, Yu CC, Su MW, Mai W, Wang HW, Cheng WC, Schuyler SC, Ma N, et al: miR-200c and GATA binding protein 4 regulate human embryonic stem cell renewal and differentiation. Stem Cell Res (Amst) 12: 338-353, 2014. 